\title{
Facilitated diffusion of glucosamine-6-phosphate synthase inhibitors enhances their antifungal activity ${ }^{\star \star}$
}

\author{
Agnieszka Janiak, Barbara Cybulska, Joanna Szlinder-Richert, Edward Borowski \\ and Sławomir Milewski ${ }^{\varpi}$
}

Department of Pharmaceutical Technology and Biochemistry, Technical University of Gdańsk, Gdańsk, Poland

Received: 10 September, 2001; revised: 7 February, 2002; accepted: 4 March, 2002

Key words: GlcN-6-P synthase, diffusion, antifungal activity, synergism, liposomes

\begin{abstract}
N3-(4-Methoxyfumaroyl)-L-2,3-diaminopropanoic acid (FMDP) and 2-amino-2deoxy-D-glucitol-6-phosphate (ADGP) are strong inhibitors of the essential fungal enzyme, glucosamine-6-phosphate synthase, but their antifungal activity is poor, due to slow penetration of these agents through the cytoplasmic membrane.

In the present studies we have exploited the possibility of enhancement of ADGP and FMDP antifungal activity by improving their transport properties. It has been found that membrane-permeabilising polyene macrolides amphotericin B (AMB) and its $\mathrm{N}$-methyl- $\mathrm{N}$-fructosyl methyl ester derivative (MF-AME), at subinhibitory concentrations, facilitate diffusion of ADGP through the fungal cell membrane, thus allowing a decrease of its minimal inhibitory concentration (MIC). Synergistic effects have been observed for combinations of ADGP with AMB or MF-AME. Fractional inhibitory concentration (FIC) indexes, determined against a number of Candida spp., have been in the 0.18-0.81 range. Weak antifungal synergistic effects have been found for combinations of FMDP with AMB or MF-AME.
\end{abstract}

\footnotetext{
${ }^{\star}$ Presented at the 8th International Symposium on Molecular Aspects of Chemotherapy, September, 2001, Gdańsk, Poland.

This work was supported in part by: the State Committee for Scientific Research (KBN, Poland), grant No. 4 P05F 00216, and Chemical Faculty of the Technical University of Gdańsk.

${ }^{凶}$ Address for correspondence: Sławomir Milewski, Department of Pharmaceutical Technology and Biochemistry, Technical University of Gdańsk, G. Narutowicza 11/12 , 80-952 Gdańsk, Poland; phone: (48 58) 347 2107; fax: (48 58) 347 2694; e-mail: milewski@altis.chem.pg.gda.pl

Abbreviations: ADGP, 2-amino-2-deoxy-D-glucitol-6-phosphate; AMB, amphotericin B; DMPC, dimyristoylphosphatidylcholine; 5-FC, 5-fluorocytosine; FIC, fractional inhibitory concentration; FMDP, $N^{3}$-(4-methoxyfumaroyl)-L-2,3-diaminopropanoic acid; GlcN-6-P, glucosamine-6-phosphate; MF-AME, $N$-methyl- $N$-fructosyl-amphotericin B methyl ester; MFC, minimal fungicidal concentration; MIC, minimal inhibitory concentration; TNBS, 2,4,6-trinitrobenzenesulfonate.
} 


\begin{abstract}
ADGP can be easily encapsulated into unilamellar lipid vesicles. Liposomal preparations of ADGP demonstrated stronger antifungal activity against some fungal strains than free ADGP.
\end{abstract}

Chitin, an important constituent of cell wall of most human pathogenic fungi, is absent from mammalian hosts. Glucosamine-6-phosphate (GlcN-6-P) synthase, an enzyme catalysing the first decisive step in the chitin biosynthesis pathway, has been proposed a promising target for antifungal chemotherapy [1]. The reaction catalysed by the enzyme is complex and involves ammonia transfer from L-glutamine amide to D-fructose-6-phosphate and subsequent ketose/aldose isomerisation of the resulting fructoseimine-6-phosphate to GlcN-6-P [2]. A number of specific GlcN-6-P synthase inhibitors are known. Most of them either are analogues of the enzyme substrate, L-glutamine, or mimic the structure of the putative transition state cis-enolamine. A glutamine analogue, $N^{3}$-(4-methoxyfumaroyl)-L-2,3-diaminopropanoic acid (FMDP), is a strong inactivator of fungal GlcN-6-P synthase [3] but a poor antifungal agent due to its slow uptake into fungal cells [4]. On the other hand, oligopeptide agents incorporating FMDP show relatively high antifungal activity, both in vitro and in vivo [5, 6]. Unfortunately, FMDP-peptides are rapidly degraded by serum peptidases [7]. Moreover, fungi can easily develop a phenotypic resistance to such peptides [8], due to the fact that peptide permeases are not essential for fungal growth [9]. Conversion of FMDP into its acetoxymethyl ester gave a lipophilic 'pro-drug', with the antifungal activity a little higher than that of the parent compound, but still unsatisfactory [4].

2-Amino-2-deoxy-D-glucitol-6-P (ADGP) was reported to be a transition state analogue inhibitor of bacterial GlcN-6-P synthase [10]. No data on its inhibitory potential against the fungal enzyme and antifungal activity have been available so far. Nevertheless, free diffusion of ADGP through the cell membrane seems very unlikely due to the presence in its structure of the charged phosphate and amino groups.

One of the possibilities of enhancement of effectiveness of antimicrobial compounds is a combination therapy. The phenomenon of synergism often enables a reduction of effective doses of chemotherapeutic agents. The only example of this approach in antifungal chemotherapy that has been applied in clinical practice is a combination of 5-fluorocytosine (5-FC) and amphotericin B (AMB) [11]. It was shown that subinhibitory doses of $\mathrm{AMB}$ facilitated diffusion of 5-FC through the fungal cell membrane [12]. Another possibility of efficient delivery into cells of non-diffusible compounds is their encapsulation into lipidic vesicles. Liposomal preparations are usually constructed in order to reduce toxicity of antimicrobial agents, and improve their bio-availability and targeted delivery into particular organs and tissues of mammalian hosts [13]. Liposomal amphotericin B could serve as a good example of a successful application of this approach in antifungal chemotherapy [14].

In the present communication we report the results of our recent studies on antifungal activity in vitro of combinations of GlcN-6-P synthase inhibitors with polyene macrolides and of their liposomal preparations.

\section{MATERIALS AND METHODS}

Micro-organisms. Clinical isolates of Candida glabrata, Candida kruzei, Candida parapsilosis, Candida famata and Candida humicola were provided by the Department of Medical Microbiology, Medical Academy of Gdańsk. Those strains and Candida albicans ATCC 10261, Candida albicans ATCC 26278 and Saccharomyces cerevisiae ATCC 9763 were stored on Sabouraud Agar slants. 
Chemicals. Amphotericin B was from Sigma. FMDP, ADGP and MF-AME were synthesised at the Technical University of Gdańsk by Dr. R. Andruszkiewicz and Dr. J. Grzybowska. All other chemicals were of the highest grade commercially available.

Susceptibility testing. Antifungal activity was determined by the serial two-fold dilution method in 96-well microtiter plates. The tests were performed in RPMI 1640 medium containing $2 \%$ glucose and buffered to $\mathrm{pH} 7.0$ with $0.165 \mathrm{M}$ Mops, strictly according to recommendations given by the National Committee for Clinical Laboratory Standards (NCCLS) [15]. The inoculum size was $10^{3}$ cells $/ \mathrm{ml}$. Plates were incubated at $30^{\circ} \mathrm{C}$ for 24 $\mathrm{h}$ and the results were read visually. Minimal inhibitory concentration (MIC) was defined as the lowest drug concentration preventing visible growth. For assessment of the fungicidal activity, $50-\mu$ l samples of solutions/suspensions were collected from each microtiter well after MIC determination. Collected samples were diluted $10^{3} \times$ with sterile saline and 0.1-ml aliquots were spread with a sterile glass rod on the surface of Sabouraud Agar plates. Plates were incubated for $24 \mathrm{~h}$ at $30^{\circ} \mathrm{C}$ and colonies were counted. Minimal fungicidal concentration (MFC) was defined as the lowest drug concentration preventing formation of fungal colonies and $\mathrm{MFC}_{90}$ as the lowest concentration causing $90 \%$ reduction of colony number in comparison to control.

Determination of the combined antifungal effect. Serial two-fold dilutions of the antifungal agents tested were prepared in microtiter plates, so that each well contained a unique combination of two agents. Their concentrations were chosen to encompass the MIC for the strains to be tested. Plates were inoculated with $10^{3}$ cells $/ \mathrm{ml}$ from overnight cultures. After $24 \mathrm{~h}$ incubation at $37^{\circ} \mathrm{C}$, plates were examined and fractional inhibitory concentration (FIC) indexes were calculated as follows: FIC index $=($ MIC of agent 1 in combination)/(MIC of agent 1 alone) + (MIC of agent 2 in combination)/(MIC of agent 2 alone). The FIC index for each row of dilutions was calculated and the greatest deviation from 1.0 used to characterise the interaction between agents.

Determination of ADGP transport rate. C. albicans ATCC 26278 cells, growing exponentially in RPMI medium, were harvested by centrifugation $(3000 \times \mathbf{g}, 5 \mathrm{~min})$, washed with $50 \mathrm{mM}$ Tris/HCl buffer, $\mathrm{pH}$ 7.0, and suspended in the same buffer containing $1 \%$ glucose, or $1 \%$ glucose and $100 \mu \mathrm{M} \mathrm{NaN}_{3}$, or no additives, to a final cell density corresponding to $\mathrm{A}_{660}=1.0$. The cell suspension was incubated at $30^{\circ} \mathrm{C}$. After $10 \mathrm{~min}$, ADGP dissolved in a minimal amount of buffer was added to give the required final concentration. At that moment and at 5-min intervals thereafter, 2-ml samples of the cell suspensions were withdrawn and immediately filtered through the Whatman GF/A filters. Portions (1 ml) of the collected filtrates were combined with $1.25-\mathrm{ml}$ aliquots of a solution containing $4 \%$ $\mathrm{Na}_{2} \mathrm{~B}_{4} \mathrm{O}_{7} \cdot 10 \mathrm{H}_{2} \mathrm{O}$ and $0.8 \mathrm{mg} / \mathrm{ml}$ of $2,4,6$-trinitrobenzenesulfonate (TNBS). The resulting mixtures were incubated at $37^{\circ} \mathrm{C}$ for $30 \mathrm{~min}$. The $\mathrm{A}_{420}$ values of the mixtures were measured and ADGP concentration in filtrates was read from appropriate standard curve. Data were plotted as nanomoles of ADGP taken up by $1 \mathrm{mg}$ (dry weight) of cells versus time. The initial uptake velocities were determined from the slopes of the linear part of the curves originating at 0.0 point.

Preparation of liposomes. A mixture of $0.3 \mathrm{ml}$ of $100 \mathrm{mM}$ dimyristoyl phosphatidylcholine (DMPC), $0.24 \mathrm{ml}$ of $100 \mathrm{mM}$ cholesterol and $0.06 \mathrm{ml}$ of $100 \mathrm{mM}$ stearylamine solutions in chloroform was further diluted with chloroform to a final volume of $3 \mathrm{ml}$. The lipid solution was combined with diethyl ether ( $3 \mathrm{ml}$ ) and $2 \mathrm{ml}$ of the $40 \mathrm{mM}$ ADGP solution in saline or with $2 \mathrm{ml}$ of saline. The mixture was sonified to form emulsion and the solvent was removed by evaporation under reduced pressure. The residue was carefully suspended in a small volume of sterile saline and liposomes were separated from free ADGP by 
gel filtration through small disposable PD-10 columns developed with saline. Fractions of 1 $\mathrm{ml}$ were collected and concentrations of ADGP and DMPC were determined in 50- $\mu \mathrm{l}$ samples withdrawn from each fraction. Fractions emerging near the void volume, containing the highest concentration of ADGP and DMPC, were combined.

Determination of DMPC concentration. Samples of fractions collected from the PD-10 column were diluted to $1 \mathrm{ml}$ with saline and combined with $1 \mathrm{ml}$ of methanol. The mixture was vortexed for $2 \mathrm{~min}, 1.5 \mathrm{ml}$ of chloroform was added and vortexing was continued for another $3 \mathrm{~min}$. The chloroform layer was carefully collected to a fresh test-tube and combined with $1 \mathrm{ml}$ of $0.1 \mathrm{M}$ ferric thiocyanate solution. The mixture was vortexed for $3 \mathrm{~min}$ and then left for several minutes for phase separation. The organic layer was collected and $\mathrm{A}_{488}$ was measured. DMPC concentration was read from the standard curve prepared for DMPC solutions in the $0-500 \mu \mathrm{g} / \mathrm{ml}$ range.

Determination of ADGP concentration. Samples of fractions collected from the PD-10 column were diluted to $1 \mathrm{ml}$ with saline containing $2 \%$ Triton X-100. Concentration of ADGP was determined by the TNBS method, essentially as described above.

Enzyme purification. C. albicans GlcN-6-P synthase was purified to near homogeneity according to the procedure described previously [16].

Determination of GlcN-6-P synthase activity. Enzymatic activity was determined by the modified Elson-Morgan procedure [17]. Reactions were carried out in $25 \mathrm{mM}$ phosphate buffer, $\mathrm{pH}$ 6.8, containing $1 \mathrm{mM}$ EDTA and $1 \mathrm{mM}$ dithiothreitol. D-Fructose-6-phosphate and L-glutamine concentrations were either fixed $(7.5 \mathrm{mM}$ and $10 \mathrm{mM}$, respectively, for determination of general activity and of concentrations inhibiting $50 \%$ of enzymatic activity, $\left.\mathrm{IC}_{50}\right)$ or variable $(0.5-7.5 \mathrm{mM}$ and 0.625-10 mM, respectively, for determinations of inhibition constants, $K_{\mathrm{i}}$. $K_{\mathrm{i}}$ values were then determined from the secondary plots of apparent Michaelis constants vs. inhibitor concentration, derived from Lineweaver-Burk plots.

Determination of GlcN-6-P synthase activity in situ. C. albicans cells, incubated for $60 \mathrm{~min}$ in $50 \mathrm{mM}$ Tris/HCl buffer, $\mathrm{pH} 7.0$, containing $2 \%$ glucose, in the presence or absence of antifungal agents, were harvested and suspended in $8.5 \mathrm{ml}$ portions of $0.1 \mathrm{M}$ imidazole/ $\mathrm{HCl}$ buffer, $\mathrm{pH} 7.0$, containing $0.2 \mathrm{M} \mathrm{KCl}$ and $0.1 \mathrm{M} \mathrm{MgCl}_{2}$, at cell density $1.2-1.6 \times 10^{9}$ cells $/ \mathrm{ml}$. Aliquots $(1.5 \mathrm{ml})$ composed of toluene/ethanol/Triton X-100, (5:20:2, by vol.), were added to the cell suspensions and the mixtures were vortexed for $5 \mathrm{~min}$ at room temperature. The cells were washed three times with $50 \mathrm{mM}$ potassium phosphate buffer, $\mathrm{pH}$ 6.8, containing $1 \mathrm{mM}$ EDTA and 1 $\mathrm{mM}$ dithiothreitol and suspended in the same buffer at $10^{8}$ cells $/ \mathrm{ml}$. L-Glutamine and Fru-6-P were added to the final concentrations of $7.5 \mathrm{mM}$ and $10 \mathrm{mM}$, respectively, and the suspensions were incubated for $30 \mathrm{~min}$ at $37^{\circ} \mathrm{C}$. Cells were removed by centrifugation and GlcN-6-P concentration was assayed in the supernatant by the modified Elson-Morgan procedure.

Other methods. Protein was determined by the Bradford procedure [18] with bovine serum albumin as a standard. Cells were counted in a Bürker chamber.

\section{RESULTS AND DISCUSSION}

The inhibitors of GlcN-6-P synthase studied in this work differ markedly as far as their chemical structure is concerned. FMDP, a glutamine analogue, was previously found to inactivate $C$. albicans GlcN-6-P synthase in a time- and concentration-dependent manner [3]. The molecular mechanism of inactivation involves formation of a covalent bond with the $\mathrm{N}$-terminal catalytic cysteinyl residue of the enzyme [19]. Inhibition by $50 \%$ of pure C. albicans GlcN-6-P synthase activity $\left(\mathrm{IC}_{50}\right)$ is 
observed at $4 \mu \mathrm{M}(1.1 \mu \mathrm{g} / \mathrm{ml})$ FMDP [3]. On the other hand, 2-amino-2-deoxy-D-glucitol-6-P (ADGP) was reported to be an inhibitor of bacterial GlcN-6-P synthase [10]. We have found that this compound inhibits homogeneous C. albicans enzyme with $\mathrm{IC}_{50}$ of $230 \mu \mathrm{M}$ $(57.5 \mu \mathrm{g} / \mathrm{ml})$. The inhibition is competitive with respect to Fru-6-P and non-competitive with respect to L-glutamine with $K_{\mathrm{i}}$ values of $35 \mu \mathrm{M}$ and $130 \mu \mathrm{M}$, respectively.

Both FMDP and ADGP are poor antifungal agents. Their antifungal activity in vitro, determined by a serial dilution method in RPMI 1640 medium, was very low. MIC values were generally in the $\mathrm{mg} / \mathrm{ml}$ range, despite the fact that the inhibitory potency of these compounds vs. their intracellular target is much higher. MIC for FMDP was $0.625 \mathrm{mg} / \mathrm{ml}$ against $C$. albicans and $S$. cerevisiae, 1.25 $\mathrm{mg} / \mathrm{ml}$ against $C$. glabrata and $2.5 \mathrm{mg} / \mathrm{ml}$ against other Candida spp. For ADGP, MIC values ranged from 2.5 to $5.0 \mathrm{mg} / \mathrm{ml}$. One of the possible reasons for such a difference between the potential to inhibit the enzyme and the antifungal activity could be a slow uptake of either compound by fungal cells. We have previously found that FMDP is taken up by C. albicans cells by an active transport system, preferably by the glutamine/asparagine transporter. Maximal uptake velocity is not higher than $5 \mathrm{nmol} / \mathrm{min}$ per $\mathrm{mg}$ dry weight and the FMDP concentration half-saturating the uptake system is $240 \mu \mathrm{M}$ [4]. Presence of nutrient amino acids in the RPMI medium obviously reduces FMDP uptake due to the competition for permease(s).

In the present work we studied the uptake of ADGP by C. albicans cells and found ADGP to be very slowly accumulated by these cells. The initial uptake velocity determined for cell suspension containing ADGP, $5 \mathrm{mg} \times \mathrm{ml}^{-1}$ (20 $\mathrm{mM})$, was as low as $1.1 \pm 0.2 \mathrm{nmol} / \mathrm{min}$ per mg dry weight. When initial uptake velocities were plotted against ADGP concentration, a hyperbolic curve was obtained (not shown), suggesting a carrier-mediated transport. From the double-reciprocal plot shown in Fig.
1 the kinetic parameters of ADGP uptake were determined, assuming a "MichaelisMenten-like" kinetics model. The maximal uptake velocity was $6.6 \mathrm{nmol} / \mathrm{min}$ per $\mathrm{mg}$ dry weight and the ADGP concentration half-saturating the carrier system was $22.6 \mathrm{mM}$. One may therefore conclude that ADGP exhibits extremely low affinity to the carrier binding site(s).

Data presented in the inset to Fig. 1 show that presence of sodium azide, $100 \mu \mathrm{M}$, in the buffer system practically stopped ADGP up-

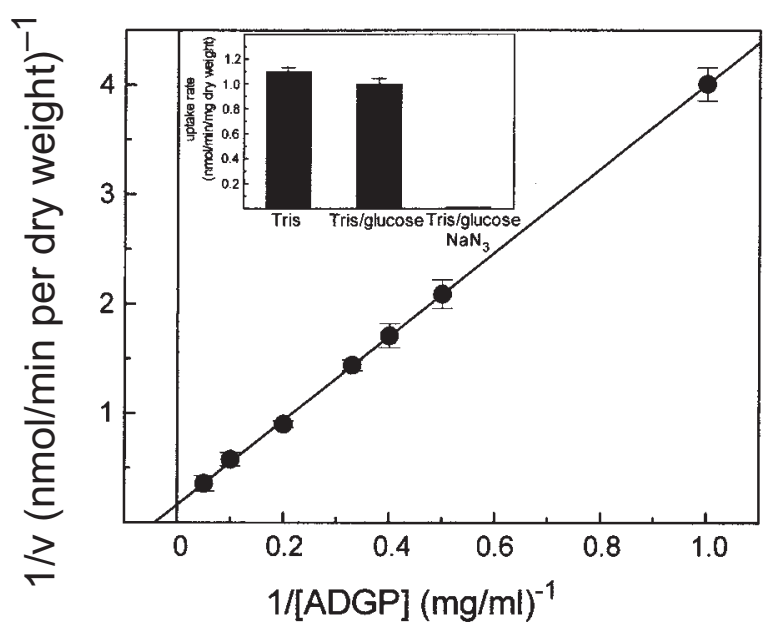

Figure 1. Kinetic analysis of ADGP uptake by $C$. albicans cells.

ADGP at different concentrations was added to the fungal cell suspensions incubated at $30^{\circ} \mathrm{C}$ in buffer solution containing $2 \%$ glucose. Samples of the suspensions were collected at time intervals, cells were harvested by filtration and ADGP concentration was determined in the filtrates. Data were plotted against time to determine the initial uptake velocities. Inset, influence of $2 \%$ glucose and $100 \mu \mathrm{M}$ sodium azide on $\operatorname{ADGP}(5 \mathrm{mg} / \mathrm{ml})$ initial uptake velocity. Values are the means of three independent determinations \pm S.D.

take. A similar effect was observed for $1 \mathrm{mM}$ sodium arsenate (not shown). This result clearly indicates that the ADGP carrier is metabolic energy-dependent. On the other hand, presence/absence of $2 \%$ glucose had no effect on ADGP uptake. It should be noted, however, that in our experiments actively metabolising cells, transferred to the buffer system and incubated there for a relatively short time were 
used. The lack of effect of glucose on ADGP uptake should not be therefore interpreted as evidence against the active transport mechanism but rather as suggesting glucose and ADGP uptake to be mediated by different carriers. Further studies are necessary to identify the actual ADGP carrier.

Assuming that the inefficient uptake could be a factor determining the poor antifungal activity of ADGP and FMDP, we have taken two approaches to facilitate their entry into fungal cells. One of them was a combined action of the inhibitors with membrane-active antifungal agents, AMB or MF-AME. Both agents demonstrate high antifungal activity in vitro. Amphotericin B is a well- known antifungal agent used routinely in clinics for the treatment of disseminated mycoses. This antibiotic binds to ergosterol and forms channels in the fungal cell membrane [20]. MF-AME is a structural derivative of AMB developed at the Technical University of Gdańsk, showing a much better chemotherapeutic index than the original antibiotic [21].

We used the checkerboard serial dilution microplate method to study the antifungal effect of combinations of GlcN-6-P synthase inhibitors with AMB or MF-AME. The results of

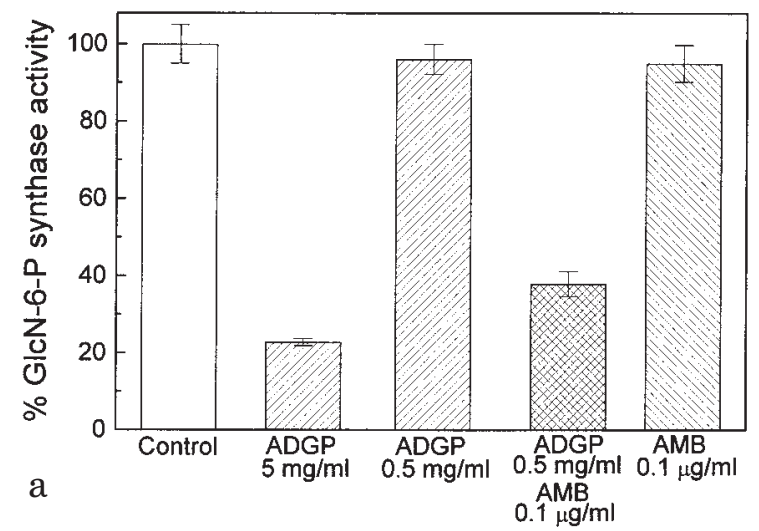

these experiments were plotted as isobolograms. Two representative graphs are shown in Fig. $2 \mathrm{a}$ and b. The values of fractional inhibitory concentrations (FIC) calculated for each experiment, as described in the Materials and Methods section, have been summarised in Table 1. FIC indexes are generally accepted quantitative measures of the extent of interaction between two agents used in combination. The effect of interaction is considered synergistic when FIC $<1$, additive for FIC $=1$, and antagonistic when FIC $>1$. Data shown in Table 1 indicate that synergism was observed for combinations of $\mathrm{ADGP}$ with $\mathrm{AMB}$ or MF-AME against all the fungal strains tested, with FIC indexes ranging between 0.18 and 0.81 . Much weaker synergism, with numerous examples of an additive effect or even antagonism, was noted for the combinations of FMDP and AMB or MF-AME.

There are three basic mechanisms of synergistic activity of chemotherapeutic agents: (a) increase by one agent of the permeability of the cell wall and cell membrane to the second agent; (b) inhibition by one agent of enzymes capable of degrading the second agent and (c) double blocking by the two components of successive steps in the metabolic sequence [22]. There is little doubt that strong synergism ob-

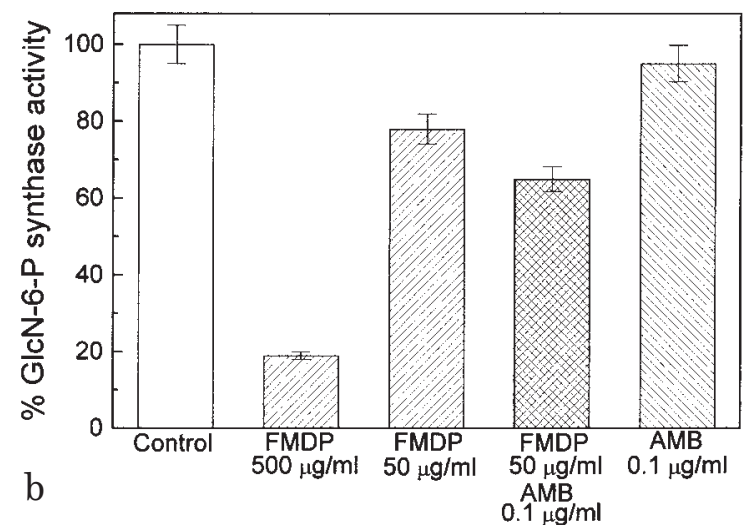

Figure 2. Inhibition of GlcN-6-P synthase activity in situ in C. albicans cells: (a) effect of ADGP, AMB and their combination; (b) effect of FMDP, AMB or their combination.

C. albicans cells incubated for $60 \mathrm{~min}$ in the presence or absence of antifungal agents were harvested, suspended in the imidazole/HCl buffer and treated with the toluene/ethanol/Triton X-100 mixture to make the cell membrane more permeable. Cells were then washed with phosphate buffer, suspended in fresh buffer and GlcN-6-P substrates were added. After $30 \mathrm{~min}$ incubation, cells were harvested and GlcN-6-P concentration was assayed in the supernatant. Values are the means of three independent determinations \pm S.D. 
Table 1. FIC indexes found for combinations of antifungal agents.

For experimental details see Materials and Methods.

\begin{tabular}{lcccc}
\hline & $\begin{array}{c}\text { AMB } \\
\text { ADGP }\end{array}$ & $\begin{array}{c}\text { MF-AME } \\
\text { ADGP }\end{array}$ & $\begin{array}{l}\text { AMB } \\
\text { FMDP }\end{array}$ & $\begin{array}{l}\text { MF-AME } \\
\text { FMDP }\end{array}$ \\
\hline C. albicans ATCC 26278 & 0.51 & 0.18 & 1.2 & 0.87 \\
C. albicans ATCC 10261 & 0.33 & 0.42 & 0.78 & 0.82 \\
S. cerevisiae ATCC 9763 & 0.29 & 0.48 & 1.0 & 0.98 \\
C. glabrata & 0.78 & 0.81 & 0.83 & 0.85 \\
C. kruzei & 0.69 & 0.58 & 1.0 & 0.78 \\
C. parapsilosis & 0.56 & 0.47 & 0.95 & 1.2 \\
C. famata & 0.73 & 0.58 & 0.96 & 1.0 \\
C. humicola & 0.72 & 0.64 & 0.81 & 0.90 \\
\hline
\end{tabular}

served for the combination of ADGP with AMB or MF-AME can be best explained by mechanism (a). ADGP alone very poorly penetrates the cell membrane and even small perturbations in the membrane structure caused by AMB/MF-AME should facilitate penetration. This assumption was confirmed by the experiment in which GlcN-6-P synthase activity was measured under in situ conditions. C. albicans cells were treated for $60 \mathrm{~min}$ with antifungal agents, processed as described in Materials and Methods and GlcN-6-P activity was determined (Fig. 3a). ADGP (5 mg/ml) alone inhibited the enzyme activity by $78 \%$, while at 10-fold lower concentration inhibition was negligible. The enzyme inhibition

a

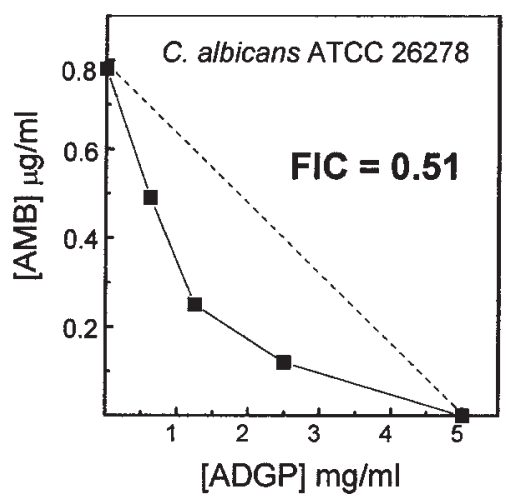

caused by ADGP at $0.5 \mathrm{mg} / \mathrm{ml}$, was substantially enhanced in the presence of AMB at $0.1 \mu \mathrm{g} / \mathrm{ml}$.

On the other hand, enhancement of GlcN-6-P synthase inhibitory potency of FMDP by AMB was much smaller (Fig. $3 \mathrm{~b}$ ), thus confirming the tendency noted from comparison of FIC indexes. Weaker amplification of the FMDP effect by AMB and MF-AME provides another evidence confirming that membrane alterations do not always facilitate uptake of poorly diffusible substances. It was previously shown, for example, that inhibition of ergosterol biosynthesis by azole antifungals had no effect on the rate of transport of a peptidic antifungal agent nikkomycin into the

b

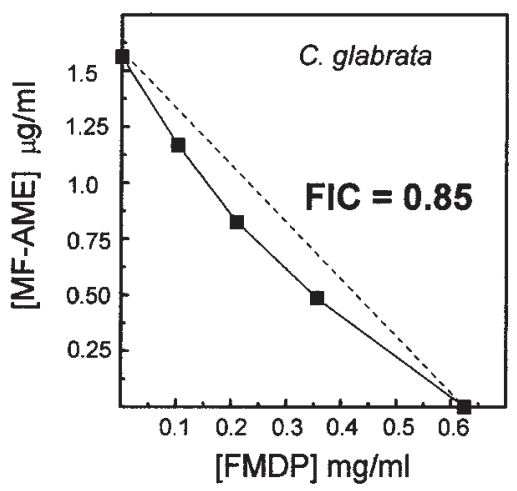

Figure 3. Exemplary isobolograms representing effects of combined action of (a) ADGP with AMB and (b) FMDP with MF-AME.

Antifungal activities of drug combinations were determined by the checkerboard serial dilution technique in the RPMI 1640 medium. FIC was calculated as described in Materials and Methods. Dotted lines represent the hypothetical additive effect. 
C. albicans cells [23]. It is therefore noticeable that FMDP's affinity to its carrier, reflected by the half-saturating concentration, is about three orders of magnitude lower than that of ADGP. Thus, although the maximal uptake velocities of the two agents are similar, FMDP is transported much faster than ADGP at low concentrations.

Taking another approach, we have elaborated a procedure of encapsulation of ADGP sual end-point detection. Antifungal activity was therefore assessed by determination of minimal fungicidal concentrations (MFC and $\left.\mathrm{MFC}_{90}\right)$.

Data presented in Table 2 show that liposomal preparations of ADGP had a slightly higher fungicidal effect than ADGP alone. The most notable reduction of MFC was found for C. albicans and S. cerevisiae. Unloaded liposomes did not affect fungal growth

Table 2. Fungicidal activity of ADGP and its liposomal preparations.

For experimental details see Materials and Methods.

\begin{tabular}{lcccc}
\hline \multirow{2}{*}{ Strain } & \multicolumn{2}{c}{ ADGP } & \multicolumn{2}{c}{ Liposomal ADGP } \\
\cline { 2 - 5 } & $\begin{array}{c}\text { MFC } \\
(\mathrm{mg} / \mathrm{ml})\end{array}$ & $\begin{array}{c}\mathrm{MFC}_{90} \\
(\mathrm{mg} / \mathrm{ml})\end{array}$ & $\begin{array}{c}\text { MFC } \\
(\mathrm{mg} / \mathrm{ml})\end{array}$ & $\begin{array}{c}\mathrm{MFC}_{90} \\
(\mathrm{mg} / \mathrm{ml})\end{array}$ \\
\hline C. albicans ATCC10261 & 10 & 10 & 5 & 0.625 \\
C. albicans ATCC 26278 & 10 & 5 & 1.25 & 0.625 \\
C. krusei & 10 & 10 & 5 & 5 \\
C. famata & 10 & 10 & $>10$ & $>10$ \\
C. glabrata & 10 & 5 & 5 & 2.5 \\
C. parapsilosis & 5 & 2.5 & 5 & 2.5 \\
C. humicola & 5 & 5 & 1.25 & 0.625 \\
S. cerevisiae ATCC 9763 & 5 & 5 & 1.25 & 0.625 \\
\hline
\end{tabular}

and FMDP into liposomes. Dimirystoylphosphatidylcholine (DMPC), cholesterol and stearylamine (molar ratio 5:4:1) were used to prepare unilamellar vesicles containing these agents. Liposomes were obtained by sonication of the lipids/agent mixture and purified by gel filtration, as described in Materials and Methods. The efficiency of encapsulation was high, so that about $90 \%$ of ADGP or FMDP molecules present in the mixtures were entrapped into liposomes. The ADGP/lipid molar ratio of the final preparations was in the $0.22-0.32$ range.

Antifungal activity of liposomal ADGP was compared to that of ADGP alone by the serial dilution microtiter method in RPMI 1640 medium. Since the presence of liposomes made the growth medium turbid, it was difficult to determine the growth inhibitory effect by vi- and viability. These results suggest that liposomal encapsulation of poorly diffusible, weak antifungal agents may improve their antifungal in vitro activity.

We are fully aware of the fact that the possibility of further development of the combinations and liposomal preparations tested by us is rather unlikely. Their antifungal activity is still too low to be of any practical value. Nevertheless, we have demonstrated that augmentation of antifungal potential of GlcN-6-P synthase inhibitors is possible and the proposed approaches may afford effective antifungal preparations, provided stronger inhibitors of GlcN-6-P synthase are found.

Skilful technical assistance of Mrs. Ewa Ortel is fully appreciated. 


\section{R E F E R E N C E S}

1. Borowski, E. (2000) Novel approaches in the rational design of antifungal agents of low toxicity. Farmaco 55, 206-208.

2. Zalkin, H. \& Smith, J.L. (1998) Enzymes utilizing glutamine as an amide donor. $A d v$. Enzymol. Relat. Areas Mol. Biol. 72, 87-145.

3. Milewski, S., Chmara, H., Andruszkiewicz, R. Borowski, E. (1985) Synthetic derivatives of $N^{3}$-fumaroyl-L-2,3-diaminopropanoic acid inactivate glucosamine synthetase from Candida albicans. Biochim. Biophys. Acta 828, 247-254.

4. Zgódka, D., Milewski, S. \& Borowski, E. (2001) A diffusible analogue of $N^{3}$-(4-methoxyfumaroyl)-L-2,3-diaminopropanoic acid with antifungal activity. Microbiology (U.K.) 147, $1955-1959$.

5. Andruszkiewicz, R., Milewski, S., Zieniawa, T. \& Borowski, E. (1990) Anticandidal properties of $\mathrm{N}^{3}$-(4-methoxyfumaroyl)-L-2,3-diaminopropanoic acid oligopeptides. J. Med. Chem. 33, 132-135.

6. Milewski, S., Andruszkiewicz, R., Zieniawa, T., Chmara, H. \& Borowski, E. (1989) Rational design of FMDP-tripeptides, a new group of antifungal agents. Proc. 16th International Congress of Chemotherapy, Jerusalem, Israel, p. 540 (1-2).

7. Kasprzak, L., Milewski, S., Gumieniak, J. \& Borowski, E. (1992) The influence of serum proteins on biological activity of anticandidal peptides containing $N^{3}$-(4-methoxyfumaroyl)-L-2,3-diaminopropanoic acid. J. Chemotherapy 4, 88-94.

8. Milewski, S., Andruszkiewicz, R. \& Borowski, E. (1988) Substrate specificity of peptide permeases in Candida albicans. FEMS Microbiol Lett. 50, 73-78.

9. Payne, J. (1986) Drug delivery systems: Optimizing the structure of peptide carriers for synthetic antimicrobial drugs. Drugs Exptl. Clin. Res. 12, 585-594.

10. Badet-Denisot, M.-A., Leriche, C., Massiere, F. \& Badet, B. (1995) Nitrogen transfer in E. coli glucosamine-6P synthase. Investigations using substrate and bisubstrate analogs. Bioorg. Med. Chem. Lett. 5, 815-820.

11. Polak, A. (1988) Combinational therapy with antifungal drugs. Mycoses 31 (Suppl. 2), $45-53$.

12. Beggs, W.H. (1986) Mechanism of synergistic interactions between amphotericin B and flucytosine. J. Antimicrob. Chemother. 17, 402-404.

13. Siler-Marinkovic, S., Mojovic, L., Davinic, V. \& Bugarski, B. (1997) Liposomes as carriers of antimicrobial drugs. Drug. Dev. Industr. Pharm. 23, 483-488.

14. Brajtburg, J. \& Bolard, J. (1996) Carrier effects on biological activity of amphotericin B. Clin. Microb. Rev. 9, 512 - 531.

15. National Committee for Clinical Laboratory Standards (1997) Reference method for broth dilution antifungal susceptibility testing for yeast: Proposed standards. Document M27-A. National Committee for Clinical Laboratory Standards. Wayne, PA.

16. Milewski, S., Kuszczak, D., Jędrzejczak, R., Smith, R.J., Brown, A.J.P. \& Gooday, GW. (1999) Oligomeric structure and regulation of Candida albicans glucosamine-6-phosphate synthase. J. Biol. Chem. 274, 4000-4008.

17. Kenig, M., Vandamme, E. \& Abraham, E.P. (1976) The mode of action of bacilysin and anticapsin and biochemical properties of bacilysin-resistant mutants. J. Gen. Microbiol. 94, 46-54.

18. Bradford, M.M. (1976) A rapid and sensitive method for the quantitation of microgram quantities of protein using the principle of protein-dye binding. Anal. Biochem. 72, 248-254. 
19. Kucharczyk, N., Denisot, M.-A., Le Goffic, F. \& Badet, B. (1990) Glucosamine-6-phosphate synthase from Escherichia coli: Determination of the mechanism of inactivation by $N^{3}$-fumaroyl-L-2,3-diaminopropionic derivatives. Biochemistry 29, 3668-3676.

20. Bolard, J. (1986) How do the polyene macrolide antibiotics affect the cellular membrane properties. Biochim. Biophys. Acta 864, 257-304.

21. Grzybowska, J., Sowiński, P., Gumieniak, J., Zieniawa, T. \& Borowski, E. (1997) N-methyl-
$N$-D-fructopyranosylamphotericin B methyl ester, new amphotericin B derivative of low toxicity. J. Antibiot. 50, 709-711.

22. Neuman, M. (1980) Basic mechanisms of antibiotic synergistic activity. Drugs Exptl. Clin. Res. 6, 259-264.

23. Milewski, S., Mignini, F. \& Borowski, E. (1991) Synergistic action of Nikkomycin X/Z with azole antifungals on Candida albicans. J. Gen. Microbiol. 137, 2155-2161. 